\section{Rules of conduct}

John A. Campbell

Benefits and Risks of Knowledge-Based

Systems. Report of a Working Party of the Council for Science and Society, chairwoman Margaret Boden. Oxford University Press: 1989. Pp. 76. Pbk £6.95, $\$ 14.95$.

ARTIFICIAL intelligence (AI) is in the news less often now than it was in the early and middle 1980s. At present there is nothing quite like the world-wide interest in the Japanese 'Fifth Generation' plan or in the novelty and first commercial successes of expert systems that produced all the previous attention. But AI research operates rather like a volcano: there is constant activity underground, which becomes visible only during eruptions. It is likely that neural nets will cause the next eruption, and in the not-too-distant future.

Among AI researchers there is the saying "AI exports its successes". What this usually means is that the wider public that makes use of the successes no longer sees them as part of AI (the languages LISP and Prolog are examples). Now expert systems seem to be going in the same direction, like lava that is cooling down

\section{THE MOTOR THEORY

$$
\begin{gathered}
\text { OF } \\
\text { LANGUAGE ORIGIN }
\end{gathered}
$$

\section{Robin Allott}

How in evolutionary terms did humans acquire language? Language cannot have sprung suddenly into existence; it must have had a precursor. The motor theory is that the precursor was the motor control system, the complex organisation of the brain for movement. The book brings together evidence from two major areas of research, linguistics and the neurology of human perception and action.

\section{£8.50 THE BOOK GUILD} 25 High St., Lewes, Sussex into easily-handled pieces of stone.

In some quarters, this comfortable trend towards cooling or domestication is even seen as not being fast enough. In the ESPRIT programme, which is an EEC response to the Fifth Generation plan, calls for proposals for research and development projects regularly contain subjectheadings such as "metrication and validation of knowledge-based systems". The implication is that computer programs built around knowledge (essentially the rule-based knowledge that is the distinguishing feature of a typical expert system) should eventually be metricated, validated and domesticated with no more trouble than for book-keeping or ticketissuing programs.

To do the writers of the ESPRIT material justice, they appreciate that they are expressing a hope rather than a certainty. It is unfortunate that this appreciation is not more widespread, especially among people who use or rely on expert-systems technology. The book reviewed here illustrates some of the issues by highlighting the present limitations of the technology, and makes suggestions about how to deal with them. It is welcome because the suggestions are modest and reasonable for example, that the systems should be treated not as oracles but as assistants to people performing tasks that require specialized knowledge, and that it should be possible for a user to examine the knowledge represented in them. But there is a risk that the book's modesty and low profile will mean that its arguments go unnoticed. Here is a rare case of a book's packaging being too sober.

The special character of knowledgebased systems (KBS) follows from the fact that knowledge is multi-faceted, openended, ambiguous and even scruffy, while the functions and specification of (say) book-keeping programs are not. Admittedly there are areas in which the relevant knowledge is well-behaved and can be given the book-keeping treatment, as in some of the most commercially successful expert systems, but these examples do not mean that all conceivable or desired KBS must have the same properties. One possible criticism of the book is that, by using the terms 'expert system' and 'knowledge-based system' almost interchangeably, it misses a chance to sharpen this point.

Clearly a KBS is founded on knowledge, in whatever representations are available to hold it. An expert system obviously has something to do with expertise, but its original meaning (most of which still survives, particularly in the United States), was tied quite tightly to one single representation for knowledge: rules. The most interesting KBS may use rules, but other representations such as frames (collections of information that belong together in some particular con- text) and inheritance nets (hierarchical structures in which specific items inherit properties from more general items, of which they are particular instances) are used at the same time. This reflects the actual organization of expert knowledge much better, and extends the range of AI applications beyond what was available in the early 1980s. But it also emphasizes the difficulty of predicting all the outcomes and pitfalls when some particular body of knowledge that goes beyond mere rules is represented and used inside a KBS. In this respect, the book gives a fair summary of the issues that deserve to be confronted, even though simple rules are the only examples of knowledge that it uses. To get the most out of the case that it makes, the reader should repeat regularly: "There is more to knowledge than rules".

The fundamental reason for the mixture of cautions and actions that the authors' collective for this book recommends is that a KBS is unlikely to use the same chain of reasoning that a human would use to solve the same problem. The methods of inference in a KBS are local approximations to our own reasoning for parts of problems - sometimes optimistic approximations (for example, the psychological evidence that we do any reasoning with the help of logic as a mathematical logician would describe it is very thin). But there is no general AI method that imitates our perception and treatment of a reasoning problem as a whole. This gives force to the recommendation that a KBS should be used as a human's assistant and not (unless provably domesticated) trusted as an autonomous agent, especially where human life and safety are concerned. An assistant who suggests initiatives should always be capable of explaining them in terms that a human senior partner can understand and accept. This concentrates attention on the ability of a typical KBS to explain its actions, and here there is still plenty of room for' improvement. Explanation, or lack of it, is also likely to be the main weakness of neural-net systems, despite their strengths in other respects. Therefore, the book's relevance will not be changed by the arrival of neural nets on the commercial scene, if this indeed causes the next eruption of publicity for AI.

Relevance and reasonableness alone are not enough to make a political impression, alas. Subversion of the established (commercial) order is a better way in 1989. If the author's subversive recommendation that a "human or group of humans ... should take moral and/or legal responsibility for the human consequences of any malfunction" of any KBS does not stir up some debate, then nothing will.

John A. Campbell is in the Department of Computer Science, University College London, Gower Street, London WC1E 6BT, UK. 